\title{
Exploring Israeli high school graduates' explanations for the spread of the coronavirus
}

\author{
Sigal-Hava Rotem ${ }^{1}$ (D) $\cdot$ Michal Ayalon ${ }^{1}$ (D)
}

Accepted: 16 February 2021 / Published online: 15 April 2021

(C) Springer Nature B.V. 2021

\begin{abstract}
The aim of this study is to explore Israeli high school graduates' mathematical explanations for the spread of the coronavirus, given that the mathematics required to do so was part of their school curriculum. An online questionnaire consisting of two sections provided a variety of potential framings for explaining the phenomenon. The first section invited the participants to explain the spread of the coronavirus in terms of their school majors in general, with no specific reference to mathematics. The second section asked explicitly to explain the mathematical context underlying the phenomenon. In this section, the participants were asked to discuss the Prime Minister's speech given in the media a few weeks earlier, in which he described the spread of the coronavirus as a geometric series. Data analysis of 87 participants' responses to the questionnaire revealed 11 different mathematical ideas used to explain the spread of the coronavirus. These ideas included are as follows: doubling, sequence, exponential growth, using powers, tree diagram, recursion, fastgrowing rate with covariation, probability, parabola and quadratic function, acceleration, and factorial. It was also found that the second section of the questionnaire elicited a wider range of mathematical ideas than the first one. We suggest possible explanations for the emergence of the mathematical ideas, which seem to reflect the graduates' intuitive knowledge, influenced not only by their mathematics track level but also by their chosen high school majors. Possible implications are discussed.
\end{abstract}

Keywords Mathematical explanations of realistic phenomenon · High school graduates' mathematical explanations $\cdot$ Exponential growth

Sigal-Hava Rotem

sigal.h.rotem@gmail.com

Michal Ayalon

mayalon@edu.haifa.ac.il

1 Department of Mathematics Education, University of Haifa, Haifa, Israel 


\section{Introduction}

Our lives have changed drastically since early 2020 due to the coronavirus pandemic. Our routines transformed overnight. While trying to cope with the radical change the virus has brought, we spend hours watching the news, following our leaders as they try to mediate the situation. In their public appearances, they try to alert us to the dangers entailed in the spread of the virus and to persuade us to follow physical distancing (which has since become obligatory).

On the $12^{\text {th }}$ of March 2020, Israeli Prime Minister (henceforth PM) Benjamin Netanyahu held a dramatic press conference in which he announced the full and immediate closure of the school system. This speech was given within the critical window of opportunity for containing the outbreak in Israel. It was the first time since the coronavirus was declared a pandemic that obligatory restrictions were imposed in Israel. Immediately afterwards, the educational sector and the majority of the business and governmental sectors were shut down, a step the consequences of which would be on the public agenda for the coming months, if not years. The PM, who was attempting to explain the necessity of these extreme restrictions, conveyed a deep sense of urgency. In order to underscore the rapid spread of the virus and the resulting need for physical distancing, the PM used the concept of a geometric series (https://www. youtube.com/watch?v=AZS5rh6QgXE starting at minute 08:43). The speech in Hebrew is followed by our free translation to English (Fig. 1):

Listening to the PM, we wondered how Israeli citizens were able to grasp his mathematical explanation, which resembled basic textbook problems encountered regularly by Israeli high school students. We were particularly interested in how those who graduated high school in recent years, who may still remember what they learned in school, explained the PM's message and what kinds of mathematical ideas they recruit to explain the spread of the coronavirus. We therefore developed an online questionnaire divided into two sections: first, we allowed participants to provide whatever explanation they deemed fitting, to see if mathematical explanations would come up spontaneously; only then did we point them in

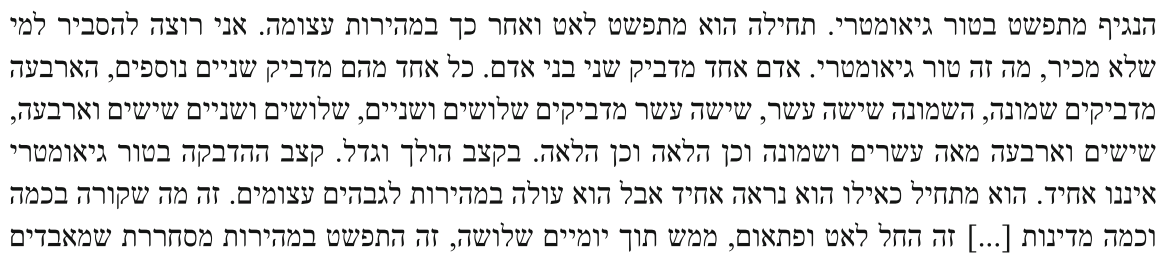

The virus spreads in a geometric series. First, it spreads slowly and then at a tremendous speed. I want to explain this to the public, which is unfamiliar with what a geometric series is. One person infects two people. Each one infects two more. Four infect eight, eight sixteen. Sixteen infect 32. Thirty-two infect 64 , sixty-four infect 128 , and so on and so forth at a growing rate. The rate of infection in a geometric series is not uniform. In the beginning, it appears to be uniform, but it quickly rises to enormous heights. This is what happened in some countries [...] It started slowly, and suddenly within two to three days, it spread at a breakneck speed that resulted in losing control.

Fig. 1 PM Mr. Netanyahu's speech given on the 12th of March 2020 (first in Hebrew followed by English) 
the direction of mathematics. In both sections, we were particularly interested in the kinds of mathematical explanations that would arise. We regard this as a unique opportunity to explore people's use of school-acquired knowledge, particularly mathematics, when attempting to interpret a phenomenon that has a significant impact on their lives.

\section{The mathematics of the coronavirus}

A prominent model used to describe the spread of the coronavirus is logistic curves (e.g., Batista, 2020; Morais, 2020; Wu et al., 2020; all preprint versions retrieved under open access). Logistic curves are used to mathematically describe the phenomenon of population growth in a constrained environment (e.g., Cramer, 2002; Lipkin \& Smith, 2001; Reed \& Pearl, 1927). Figure 2 illustrates population growth under constraint $\mathrm{k}$ (taken from Reed and Pearl (1927)).

What the logistic curve tells us about the spread of the coronavirus is that over time, an increasing number of people becomes infected. Each day, the number of new cases exceeds the number of new cases on the preceding day. The coronavirus spread is constrained by the number of people in the general population who have the potential to get infected. The trend of the increased rate of change goes up until the virus does not "meet" enough new people to infect; thanks to a vaccine, natural immunity, or physical distancing measures. At that point, the logistic curve has an inflection point, after which the daily number of newly infected people begins to decrease. Figure 2 presents the curves both for the total number of infected people (the logistic curve) and the rate of change in the new cases (the first derivative curve).

One of the mathematical ideas comprising the Israeli school curriculum and underlining this phenomenon is covariation - "two or more quantities varying simultaneously" (Thompson \& Carlson, 2017; p. 423) - which is apparent both in the logistic curve, where the number of infected people changes simultaneously with time, and in the curve's first derivative, where the

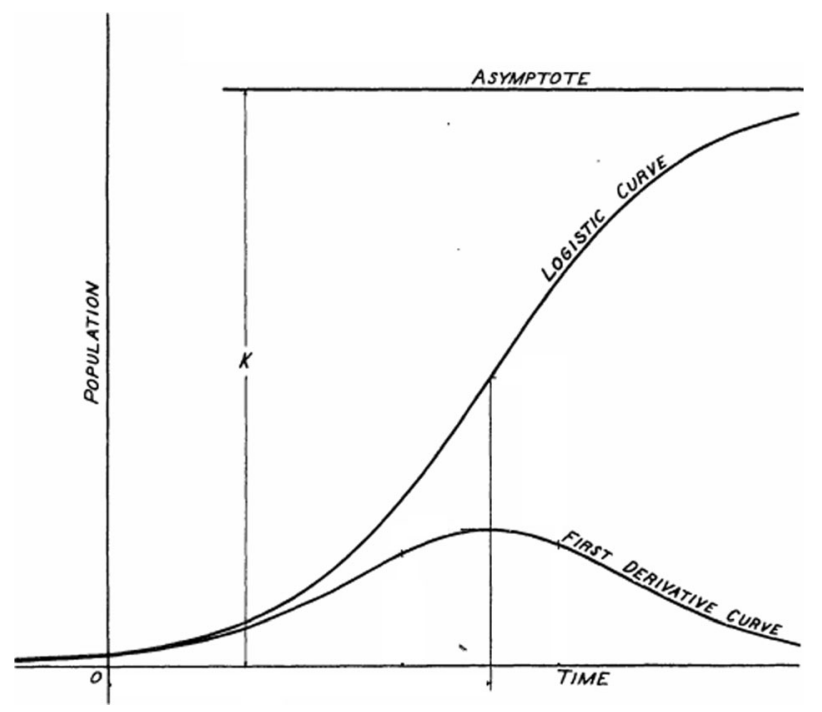

Fig. 2 Logistic curve and its first derivative curve (Reed \& Pearl, 1927) 
number of new cases changes simultaneously with time. Second is the idea of constrained growth, which results in an inflection point in the logistic curve and extremum in the first derivative, after which there is a decreased rate of change. Third is the idea of exponential growth, which describes the unconstrained natural growth up to the inflection point (further elaboration can be found in Lipkin and Smith's (2001) paper).

The mathematical ideas underlying this model will serve us as a backdrop against which we will interpret participants' explanations. Returning to the PM's speech, which aimed to convey the rapid spread of COVID-19, we see that it focused heavily on the mathematical idea of exponential growth, using the representation of a geometric series. The mathematical ideas that appear in his speech can be categorized into ideas mentioned explicitly in the numerical example, mathematical ideas implied by the numerical example, and those implied by the wording used.

The geometric series idea was explicitly mentioned and demonstrated using the numerical example "...one person infects two people. Each one infects two more. Four infect eight... and so forth...." This example implicitly suggests the ideas of sequence and using powers. Sequence, which can be regarded as a generalization of the "jumps" within the numerical example, also implies the idea of using powers to articulate the sequence pattern. Using powers is an advanced version, a generalization, of the idea of repeated doubling (Ellis et al., 2016). These ideas were not mentioned explicitly in the PM's speech but could be inferred from the numerical example he used.

The mathematical ideas implicitly suggested by the wording of the speech are exponential growth, acceleration, and growing rate with covariation. The idea of exponential growth could be inferred from the PM's use of the words "geometric series." Additionally, by saying “... suddenly within two to three days," the PM addressed two quantities that change simultaneously - the number of infected people and the time - which could imply the mathematical idea of growing rate with covariation. Furthermore, the PM's words "First it spreads slowly and then at tremendous speed..." could imply the idea of acceleration as it appears in everyday language (and not in the sense of the change in velocity).

\section{Exponential growth in the Israeli curriculum}

The premise of this study is that the fast-growing rate of spread of the coronavirus, up to the inflection point, can be explained by the mathematics included in the Israeli curriculum. Exponential growth is taught as part of the Israeli secondary school curriculum. It is part of the matriculation exams (at the end of high school) for students at all levels of study (advanced, intermediate, and basic). The issue of exponential growth is often discussed through realistic contexts related to four kinds of phenomena: (a) loan payments, (b) population growth, (c) yeast growth, and (d) viruses and bacterial growth. For example, a commonly used textbook problem, presented in Fig. 3, refers to a bacterial culture in which each bacterium divides into two every half hour. This problem, which is reminiscent of the task of making sense of the spread of COVID-19, is typical of problems which students encounter during their studies.

When learning exponential growth in a realistic context, the basic exponential equation that is addressed in Israeli schools is $M_{t}=M_{0} \cdot q^{t}$. In this equation, $q$ represents the rate of growth, $t$ is time, $M_{0}$ is the amount at the outset, and $M_{t}$ is the amount when the time is $t$. In the context of the coronavirus, the number of infected people can be calculated at a specific time, depending on the rate of growth, which may vary between countries. In the Israeli PM's 
In a bacterial culture, each bacterium divides into two for half an hour.

At noon there were 30,000 bacteria in the culture.

A. How many bacteria were cultured at 18:00 pm?

B. How many bacteria were cultured earlier that morning at 10:00 am?

Fig. 3 A sample question taken from the basic-track level (Geva \& Tal, 2014, p. 185)

explanation, the rate of growth is two: in every time frame, the number of infected people is multiplied by two. At the outset, the number of infected people is one, which yields the following equation: $M_{t}=2^{t}$. Understanding the growth of the two quantities of the equation can be done inductively, as the PM demonstrated in his speech, and similarly to the calculations which students are regularly required to perform, as demonstrated in the example (Fig. 3). We therefore suggest that the current events can be explained (at least in a basic way) using school mathematics.

\section{Teaching and learning "exponential growth"}

The problem presented above, which is characterized by the idea of repetitive multiplication by two, is typical when learning about exponential growth in other curricula as well (e.g., Confrey \& Smith, 1995; Doerr, 2006; Ellis et al., 2016; Webb et al., 2011). This contextual problem resonates with the realistic mathematics education (RME) approach to teaching and learning mathematics (Van den Heuvel-Panhuizen \& Drijvers, 2014). RME is characterized by "rich, 'realistic' situations [that] are given a prominent position in the learning process." (ibid; p. 521). The majority of the research that addresses understanding and possible difficulties while learning exponential growth is positioned in the RME context (e.g., Ellis et al., 2016; Webb et al., 2011).

Ellis et al. (2016) identified students' three learning trajectories for exponential growth: prefunctional reasoning, the covariation view, and the correspondence view. Pre-functional reasoning is a qualitative understanding of exponential growth, whereby students describe growth as being slow at the beginning and becoming faster over time. The students understand that one of the axis values grows larger when increasing the other axis value; however, the increments are not quantified. The second trajectory is the covariation view, in which "students can coordinate the change in $y$ values for multiple unit changes in $x$ values, but their mental imagery is grounded in the actions of repeated multiplication" (p. 164). The third trajectory is correspondence reasoning, where students express the repeated multiplication pattern for a growth factor $q$ algebraically as $y=q^{x}$ and understand the growth factor effect.

Confrey and Smith (1995) suggested a teaching approach that uses a multiplicative operation of "splitting" to introduce the exponential as a concept. They argued that the "splitting" operation better describes some multiplication situations, for example, in the case of a tree diagram that is a representation of recursive thinking. Davis's (2009) research provides evidence for the deficiency in seeing exponential growth as repeated multiplication. He shows that prospective secondary teachers' understanding of exponential growth as repeated multiplication does not promise their understanding of the generalized equation. Another difficulty that can emerge in understanding exponential growth equations relates to covariation of the growth. While some students may imagine the change of the two quantities 
as a smooth progression, others may imagine the change of the two quantities in completed "chunks," which is a discrete kind of thinking (Castillo-Garsow, 2012; Castillo-Garsow et al., 2013; Thompson, 2011).

These studies focus on learning and teaching the concept of exponential growth. The focus of the present study is different; here, we focus on the kinds of explanations given by graduates for the spread of the coronavirus. We are not concerned with processes of learning in school, but rather, with the school-acquired knowledge that graduates recruit when making sense of a phenomenon. Still, the above studies will serve us in interpreting the mathematical ideas raised by the research participants, as well as in offering some possible future implications for research and practice.

\section{Explaining the world through mathematics}

Several researchers have examined the mathematics which people use outside the formal context of school. For example, in their seminal work, Carraher et al. (1985) demonstrated that children who work as street vendors in Recife in Brazil had "difficulty with routines learned at school and yet at the same time [were] able to solve the mathematical problems for which these routines were devised in other more effective ways" (p. 21). Another example is Hoyles et al.'s (2001) study, which examined the use of mathematics outside a school context by adults and found that experienced nurses used a range of correct proportional-reasoning strategies, rather than the method they learned during their training.

In this study, we focus on the ways the participants explain a phenomenon using their schoolacquired mathematical knowledge. Kapon (2016) offered three dimensions for analyzing students' explanations for physical phenomena: intuitive knowledge, the mechanism, and the framing. The construct of intuitive knowledge, which is based on previous work by Fischbein (1987) and Kapon and diSessa (2012), refers to the content of the explanation; "accounts of how things are, but apart from our experience with the world, their source can also be education, language, and interaction with other people; they can be encoded both verbally and nonverbally, and they can have different levels of complexity." (p. 169). The mechanism is a moment-tomoment description of how things work (diSessa, 1996), and the framing concerns the way in which the context of the request for explanation influences the explanation itself. Kapon's (2016) study operationalized each dimension and explained through this framework the way two seventh-grade girls who attempted to understand why a plastic bottle shrinks when air is pumped out of it, negotiate their explanations and reach consensus.

We found Kapon's (2016) framework useful for the purposes of our study. The construct of intuitive knowledge allowed us to consider the different explanations that were given. The construct of mechanism allowed us to "zoom in" on the elements of the mathematical explanations. In some explanations, we identified intuitive knowledge or the mechanism, while in others, we identified both. Since we were interested in the types of mathematical explanations that participants used, we decided not to distinguish between intuitive knowledge and mechanism, as they both make up an explanation. Moreover, the construct of framing allowed us to analyze each explanation in relation to the questionnaire section in which it emerged, i.e., whether with or without an explicit reference to school mathematics. Building on Kapon's (2016) framework, in this study, an explanation for the spread of the coronavirus consists of a mathematical idea that accounts for "how things are and how they work," and is 
framed in the specific context of the request for an explanation (i.e., with or without an explicit reference to school mathematics). Our research question is as follows: what explanations do Israeli high school graduates give for the spread of the coronavirus, given that the mathematics required to do so was part of their school curriculum?

\section{Methodology}

\subsection{Research participants and setting}

Since this study aims to explore Israeli high school graduates' mathematical explanations for the spread of COVID-19, a few words about the Israeli education system are in order. Students in Israel are placed in a level of mathematics (basic, intermediate, and advanced) depending on their mathematics achievements, and select two high school majors. These majors could include social sciences (e.g., sociology, psychology, and economics), STE (e.g., STEM subjects excluding mathematics, including physics, chemistry, biology, and computer science), and the arts and humanities. The majority of Israeli graduates do not continue their education directly after high school, mainly due to compulsory military service with a minimum of 2 years for females and 3 years for males. Therefore, we decided that research participants would be no more than 5 years past their graduation. It seems a reasonable time period for potentially retaining what was learnt in school, while largely assuming that participants did not use their mathematical knowledge they had gained out of school (in higher education, for example).

The research was conducted using semi-open questionnaires, which were constructed in Google forms and distributed through the WhatsApp application in a manner that resembled virtual snowball sampling (Baltar \& Brunet, 2012). The first author sent the questionnaire to her former students (taught by her in the 2018-19 academic year) and asked them to pass it on to their peer group. Additionally, the first author asked her colleagues to send the questionnaire to their former students with the same request that they pass it on. We also distributed the questionnaire through teachers' WhatsApp groups. This form of distributing the questionnaire had some disadvantages in that participants wrote their answers via their mobile phones, which was liable to result in quick and inexact answers. Nevertheless, we chose this form of distribution as a means to reach as many participants as possible, since the WhatsApp application is a common communication channel in Israel, especially in our target group of participants (Tang \& Hew, 2017).

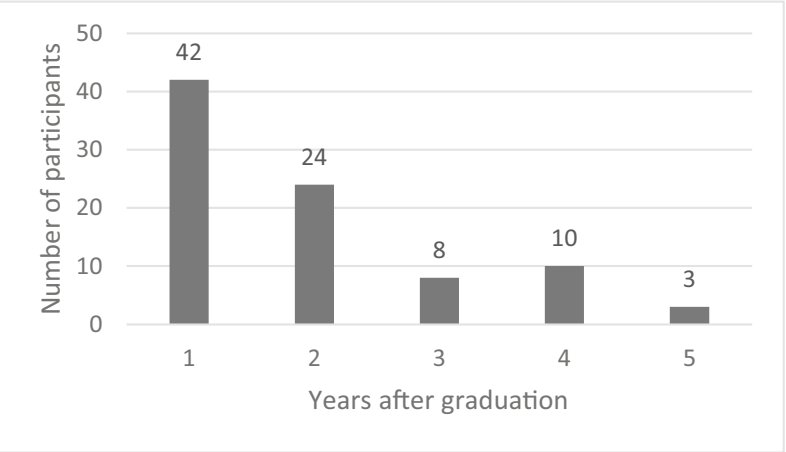

Fig. 4 Number of participants vs. the years that have passed from high school graduation 


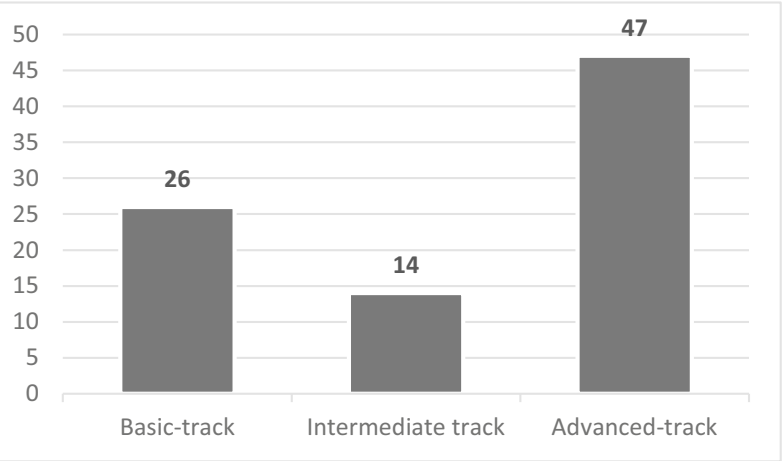

Fig. 5 Number of participants vs. the level at which they study mathematics

In total, out of 101 respondents, 14 were excluded; most of them had exceeded 12 years since their high school graduation. Therefore, 87 research participants were left. The average time that had elapsed since graduation was nearly 2 years. For about half of them, 42 out of 87 , the time lapse was 1 year. For 24 participants, the time lapse was 2 years. For the 21 remaining participants, the time that had passed since their high school graduation was 3 to 5 years. Figure 4 presents the number of participants per number of years that passed since high school graduation.

Forty-seven of the 87 participants studied in the advanced mathematics track in high school, 14 in the intermediate track, and 26 in the basic track (see Fig. 5). Twenty participants had been biology majors, 21 social science majors, 56 STE majors (here considered as physics, chemistry, and computer science), and 46 had been art and humanities majors (in Israel, a student usually has two majors).

\subsection{The questionnaire}

This study aimed to explore the kinds of mathematical explanations for the spread of the coronavirus suggested by high school graduates, both with and without an explicit instruction to refer to school mathematics. In the introduction of the questionnaire, we stated the following goal: "To learn about how you explain the spread of the corona through topics learned in high school." In this opening remark, we did not direct participants to mathematics, due to our interesting in seeing whether mathematical explanations would emerge spontaneously. Then, the questionnaire was split into two main sections offering different framings (Kapon, 2016) for explaining the spread of the virus, the first of which had to be completed to progress to the next. The first section contained an open question: "Can you explain the spread of the coronavirus with regard to something you learned in high school? If so, what subject is it, and how does it explain coronavirus's spread?" The purpose was to frame the explanation within the school curriculum, so as to learn what school-acquired knowledge the participants recruited in order to explain the phenomenon, with no restriction to mathematics. The second section of the questionnaire invited respondents to make sense of the specific part of the PM's speech that described the spread of the coronavirus as a geometric series. The aim was to frame the explanation for the spread of the virus within school mathematics. We used the PM's speech as an artifact to stimulate thought because the speech was broadcast on all Israeli media networks, and the nation's eyes were focused on it. PM Netanyahu's words played a significant role in conveying the critical importance of physical distancing. His choice to use 
Table 1 The structure and contents of the questionnaire (the English version is followed by Hebrew)

\begin{tabular}{|c|c|c|}
\hline Framing & Questions & Purpose \\
\hline $\begin{array}{l}\text { A: High school } \\
\text { curriculum }\end{array}$ & 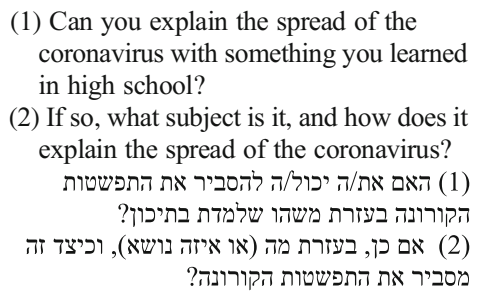 & $\begin{array}{l}\text { To link the spread of the coronavirus to the high } \\
\text { school curriculum. We wanted to ascertain } \\
\text { which mathematical ideas, if any, emerge } \\
\text { spontaneously, while taking into account that } \\
\text { some participants may address school-related } \\
\text { though nonmathematical explanations. }\end{array}$ \\
\hline $\begin{array}{l}\text { B: High school } \\
\text { mathematics }\end{array}$ & 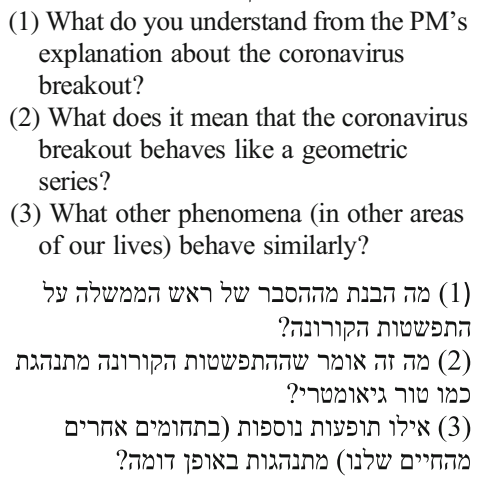 & $\begin{array}{l}\text { In this section, the PM's speech served as an } \\
\text { artifact to prompt participants to connect the } \\
\text { coronavirus outbreak to mathematics. }\end{array}$ \\
\hline
\end{tabular}

mathematics to emphasize the severity of the situation was quite remarkable. We saw an opportunity to explore the facets of mathematics his speech sparked among school graduates. "Optimistic" expectations would be that participants would refer to their knowledge of exponential growth, either descriptively or literally, by articulating that the spread of the virus can be modeled using this mathematical concept. However, we were open to exploring any other ideas that might emerge, in either of the questionnaire sections. Participants were also asked background questions about their current occupation, the level (advanced, intermediate, or basic) at which they had studied mathematics in high school, and the subjects they had majored in. We deemed this information a possible source for interpreting the results. The structure and contents of the questionnaire are presented in Table 1. Note that the questionnaire was in Hebrew, and all relevant parts of the questionnaire presented here were translated to English by us.

\subsection{Data analysis}

Overall, each of the 87 participants was asked to provide two explanations for the spread of the coronavirus. One was framed within general high school knowledge without restricting it to mathematics, and the other was framed explicitly within school mathematics. We received 174 explanations (including cases in which a participant wrote that they had no explanation). Data analysis comprised three phases, presented in Fig. 6 and elaborated below.

First, the explanations were sorted according to their fields of reference, yielding four categories: biology-based explanations, social science-based explanations, mathematics-based 


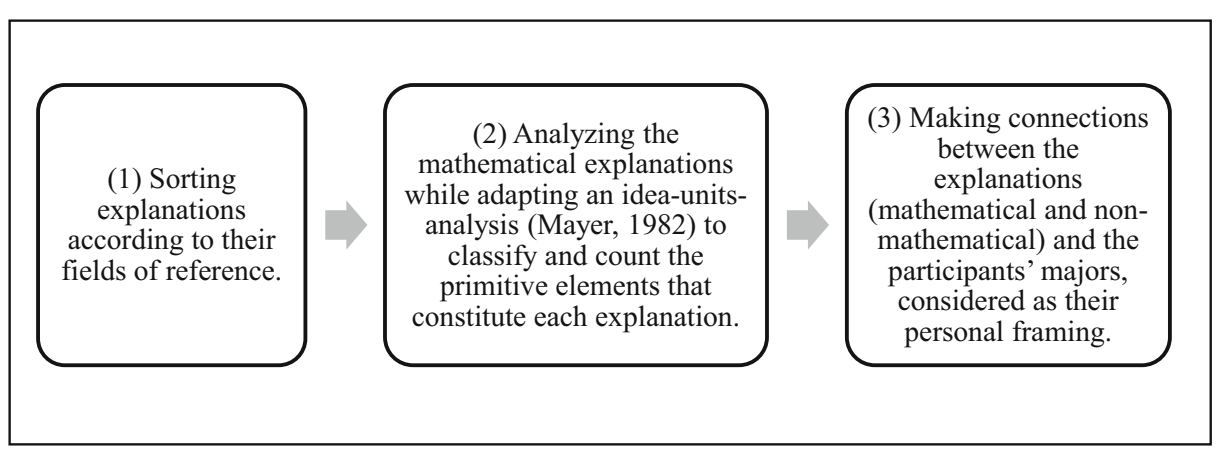

Fig. 6 Three phases of data analysis

explanations, and no explanation. For example, P33 (P stands for participant) used a social science-based explanation in the first section of the questionnaire.

I can explain it through the concept of globalization, which I learned in civics lessons, that describes the increasing transport and movement in the past years [...] between the different countries [...]. The idea of globalization can explain the rapid spread of the coronavirus, the movement of people from one country to another which spread the virus in the world $[\ldots]$.

In this explanation, P33 emphasized the concept of globalization and mentioned that he had learned it in civics lesson; both the concept and the lesson are a part of social science. Notably, there is no reference to mathematics in this explanation.

The second phase of the data analysis focused on the mathematical explanations ( 94 out of 174). This phase of analysis was implemented by adapting an idea-unit analysis to examine the kinds of mathematical ideas used, in either of the two questionnaire sections. Idea-units were defined by Mayer (1982) and employed in several later studies (e.g., Ayalon \& Hershkowitz, 2018). Idea-units are the primitive elements that constitute explanations in a questionnaire's items; their distinctiveness enables clear-cut classification and counting. In this study, explanations were divided into idea-units, each representing a distinct mathematical idea. For example, when responding to the second section, P45 explained that "it spreads [...] when each person infects two people, so it creates an equation that looks like this: $y=x^{2}[\ldots]$." This explanation includes two idea-units: the first relates to "doubling": "when each person infects two people," and the second relates to quadratic function: "it creates an equation that looks like this: $y=x^{2}$." For our purposes - exploring what kind of mathematical ideas the participants used when explaining the spread of the coronavirus - the question of whether or not the explanation was correct was irrelevant, and here, we could distinguish between two ideas: "doubling" and quadratic function. Another example is P20's response: "the spread of the illness in geometric series, meaning it is like a sequence where each element is multiplication by two of the previous term. So, the virus might infect a very large number of people...." P20 provides two idea-units. The first sequence suggests the following: "the spread of the illness in geometric series, meaning it is like a sequence," and the second is doubling "each element is multiplication by two of the previous term." Overall, the analysis of the total 94 mathematical explanations in both sections revealed 127 idea-units, where an idea-unit was counted once per participant (if the same participant addressed the same idea in both framings, it was counted as one idea-unit). 
The 127 idea-units reflected 11 different types of mathematical ideas: doubling (repeated multiplication by two), sequence, exponential growth, using powers, tree diagram, recursion, fast-growing rate with covariation, probability, parabola and quadratic function, acceleration, and factorial. Descriptions, examples, and frequencies for the 11 categories are given in the following section.

In the third phase of the analysis, the aim was to explore possible connections between the explanations (mathematical and nonmathematical) and their framings. Thus, a distinction was made between explanations given in the first section of the questionnaire, where there was no specific reference to school mathematics, and those given in the second section, which referred explicitly to school mathematics. A connection was then made between these explanations and the participants' majors, which can be considered their personal framing.

\section{Findings}

This section comprises four parts. The first part presents the collection of 11 different mathematical ideas that were used by the school graduates to explain the spread of the coronavirus. The second and third parts focus on the differences found in the number (part 2) and variation (part 3) of the mathematical explanations between the two framings (i.e., the first and second sections of the questionnaire). The fourth part describes the relations between the participants' explanations and their school backgrounds.

\subsection{The collection of the participants' mathematical explanations}

Data analysis of the high school graduates' explanations of the spread of the coronavirus, in response to the two questionnaire sections, revealed 127 idea-units, which reflected 11 different mathematical ideas. Table 2 presents these ideas, accompanied by descriptions and examples taken from the graduates' responses.

As shown in Table 2, the mathematical idea of doubling was the most common and appeared 40 times. Doubling, which is a more primitive notion of powers (Ellis et al., 2016), is connected directly to the PM's numerical example: $2,4,8,16,32,128, \ldots$. The next relatively common idea (appearing 22 times) was exponential growth, mentioned in the literature review section as the most appropriate model to use in this case. The concept of sequence appeared 18 times. This idea can be seen as a generalization of the "jumps" within the numerical example used in the PM's speech.

The other eight ideas appear more sporadically. The notion of using powers was repeated eight times. The ideas of the tree diagram and recursion (mentioned six and four times, respectively) appear in the literature dealing with exponents as representations of an exponential function (Confrey \& Smith, 1995). The idea of a growing rate with covariation (mentioned five times) is a milestone in learning exponential growth (Ellis et al., 2016).

Probability, mentioned five times, can be seen as an additional explanation. It suggests an additional tier in the model for the spread of the virus, since when an infected person meets another person, there is a probability that they will infect that person. The ideas of parabola/quadratic function and acceleration (in the sense of the rate of change of the velocity) are interesting ideas to use in this case. We would suggest that the PM's words, "First it spreads slowly and then at tremendous speed..." seem to imply acceleration. Furthermore, the examples used “... One person infects two people. Each one infects two more. Four infect eight...," which represent doubling, might be confused with or articulated as a quadratic function/parabola. Davis's (2009) work 
Table 2 Idea-units in explaining the coronavirus spread

\begin{tabular}{llll}
\hline Mathematical idea & Description & $\begin{array}{l}\text { Example (P refers to } \\
\text { index of participant) }\end{array}$ & $\begin{array}{l}\text { \# of } \\
\text { appearances } \\
(N=127)\end{array}$ \\
\hline
\end{tabular}

Doubling

Exponential growth

Sequence

Acceleration

Parabola/quadratic function

Using powers

Tree diagram

Growing rate with covariation

Probability

Recursion

Factorial
Participants explain the spread of the coronavirus as being doubled or multiplied by two, over time.

Participants refer to exponential growth as a concept that describes the spread of the coronavirus.

Participants refer to sequence as a concept that describes the spread of the coronavirus.

Participants refer to acceleration as a concept that describes the spread of the coronavirus.

Participants refer to the quadratic function or parabola as a concept that describes the spread of the coronavirus.

Participants explain the spread of the coronavirus using the concept of powers.

Participants refer to a tree diagram as a concept that describes the spread of the coronavirus.

Participants explain the spread of the coronavirus by referring to the growing rate of the number of infected people as changing over time.

Participants explain the spread of the coronavirus using probability.

Participants refer to recursion as a concept that describes the spread of the coronavirus.

Participants refer to factorial as a concept that describes the spread of the coronavirus.
"It means that the spread is doubled 40 [...], and therefore it is getting bigger." (P55)

"Each one infects few people and so 22 the growth is exponential." (P42)

"The number of infected is a geometric sequence, although I think it is more like a Fibonacci sequence because not all who get the virus is sick in a way that they can get other people infected." (P16)

"It gains more and more acceleration 10 until it cannot be controlled." (P57)

"The corona spreads like a quadratic 8 function." (P5)

"According to what I understand, the rate of the spread looks like the graph of the function of $y=x^{2}$." (P77)

"The rate of infection grows very fast 8 because the equation has a power, so as the power gets larger, the infection rate grows." (P52)

"The virus spreads like a binary tree...." (P14)

"As times goes by, then the rate of the spreading grows, and more people get infected in each given moment." (P2)

"The more infected people there are, 5 the greater the chance of getting infected." (P75)

"Recursion - I do not remember how 4 it is connected or what is exactly the recursion method, simply this is what came to my mind." (P22)

"It also a bit reminds me factorial in 1 mathematics" (P49)

indicated that even prospective secondary-school teachers struggle with the power properties of exponents. This could possibly account for these participants' explanations which confused doubling with parabola/quadratic function. 


\subsection{Mathematical ideas as dependent on framing}

The data analysis indicated that the second questionnaire section, in which participants were asked to explain the PM's speech in which he described the spread of coronavirus as a geometric series, spurred more mathematical explanations than the first framing, in which participants were asked to explain the spread of the coronavirus in terms of their school studies in general, with no specific reference to mathematics.

In the first context, 16 of the 87 participants used mathematical explanations, even when not urged to, while 71 did not. Out of these 71 participants, 36 used biological or sociological explanations, with no reference at all to mathematics, and the remaining 35 provided no explanations at all. In the second section, 73 participants gave mathematical explanations, while the remaining 14 participants provided no explanation at all. Figure 7 presents the number of participants that used mathematical or nonmathematical explanations in the first and second framings.

For example, P2 used a biological explanation in the first framing (see Table 3), trying to connect the spread of the pandemic to topics he had learned in biology class. In the second framing, P2's explanation, though incorrect, was mathematical and included three idea-units. He had the correct notion of a fast-growing rate with covariation as he was trying to grasp the change in two quantities - people getting infected and time. Additionally, P2 compared the spread of the virus to a parabola, perhaps because of the doubling idea that he later raised or a misunderstanding of the role of power in exponential growth (Davis, 2009).

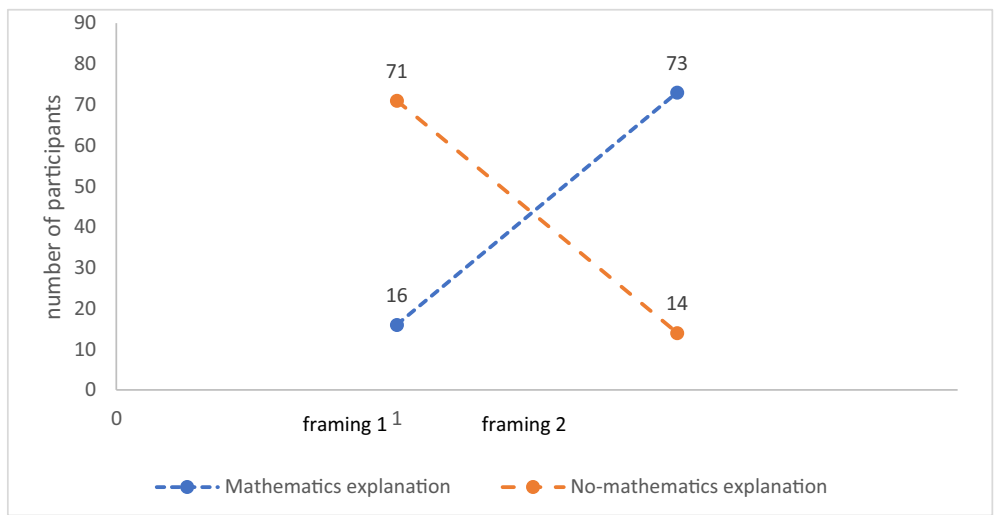

Fig. 7 Number of participants who used mathematical or nonmathematical explanations in the $1^{\text {st }}$ and $2^{\text {nd }}$ framings

Table 3 P2's explanations for the spread of the coronavirus

\begin{tabular}{|c|c|}
\hline Framing & P2's explanations \\
\hline $\begin{array}{l}\text { High school } \\
\text { curriculum }\end{array}$ & $\begin{array}{l}\text { "The virus (like any other virus) is, in fact, a microscopic creature made up of hereditary } \\
\text { material and proteins. It is not a living creature since it can only reproduce when it } \\
\text { penetrates the cell of a living creature." }\end{array}$ \\
\hline $\begin{array}{l}\text { High school } \\
\text { mathematics }\end{array}$ & $\begin{array}{l}\text { "As time goes by, the rate of the spread grows, and more and more people that are infected } \\
\text { at every minute... it is like a parabola, where the number of infected is multiplied by } \\
\text { two as it goes to another person." }\end{array}$ \\
\hline
\end{tabular}


Table 4 P26's explanations for the spread of the coronavirus

\begin{tabular}{ll}
\hline Framing & P26's explanations \\
\hline $\begin{array}{l}\text { High school } \\
\text { curriculum }\end{array}$ & "No." \\
$\begin{array}{c}\text { High school } \\
\text { mathematics }\end{array}$ & $\begin{array}{l}\text { "The spread of the virus looks slow, in the beginning, but gradually the rate of the spread } \\
\text { increases. Because of the number of infected, the more infected, there are, the more } \\
\text { options the virus has to infect... when there is one infected person who infects two } \\
\text { people, and each one of them [infects] another two and each one of them [infect] } \\
\text { another two. In this way, for example, the virus is carried and reaches } 128, \text { and each one } \\
\text { infects two, so it becomes } 256 \ldots \text { in the beginning, it looks linear, but in fact, it is } \\
\text { parabolic..." }\end{array}$ \\
\hline
\end{tabular}

Another example of the difference in responses between the two framings is P26's answer (presented in Table 4). Here, no explanation is given in the first framing, followed by a detailed explanation in the second framing in which P26 proposed the idea of doubling: "one infected person infects two people and each one of them [infects] another two and each one of them [infects] another two." After explaining the doubling idea, P26 added the idea of a parabola.

The above examples illustrate the differences in the number of mathematical explanations given in the first framing compared to the second. In addition, we found differences in the variation of mathematical explanations between framings, as described in the following section.

\subsection{The variation of mathematical ideas corresponding to their framing}

In the second framing, there were not only more mathematical explanations, but also more variation within those explanations. Table 5 presents the type and number of mathematical ideas in each framing.

As shown, in the first framing, 18 mathematical idea-units of five types emerged. In the second framing, there were 109 idea-units of 11 types.

An example of the wider variation of ideas appearing in the second framing is the case of participant P77, presented in Table 6. P77 used a mathematical explanation comprising two

Table 5 The mathematical ideas that emerged in each framing

\begin{tabular}{lll}
\hline & Frequency & \\
\cline { 2 - 3 } & First framing & Second framing \\
\hline Doubling & 0 & 40 \\
Using Powers & 0 & 8 \\
Sequence & 3 & 15 \\
Exponential Growth & 9 & 13 \\
Acceleration & 0 & 10 \\
Growing rate with covariation & 0 & 5 \\
Tree Diagram & 2 & 4 \\
Recursion & 0 & 4 \\
Probability & 2 & 3 \\
Parabola / Quadratic function & 2 & 6 \\
Factorial & 0 & 1 \\
N = & 18 & 109 \\
\hline
\end{tabular}


Table 6 P77's explanations for the spread of coronavirus

\begin{tabular}{|c|c|}
\hline Framing & P77's explanations \\
\hline $\begin{array}{l}\text { High school } \\
\text { curriculum }\end{array}$ & $\begin{array}{l}\text { "Probability, meaning the chance that a person will get sick, and the number of sick people } \\
\text { will grow from one person that came in touch with him/her. It is like a binary tree } \\
\text { diagram..., one person infects two others, and the percentage of recovery also has an } \\
\text { influence." }\end{array}$ \\
\hline $\begin{array}{l}\text { High school } \\
\text { mathematics }\end{array}$ & $\begin{array}{l}\text { "The spread of the coronavirus is not uniform but dynamic, meaning one moment it looks } \\
\text { uniform, but at some point, it gains acceleration and raises like a function without a } \\
\text { limit. But I believe that this function at some point will have an extremum point and that } \\
\text { the function will shift direction, and then the percentage of sick people will decrease in } \\
\text { the same speed at which it increased... I understand that the rate of the spread looks like } \\
y=x^{2} . "\end{array}$ \\
\hline
\end{tabular}

mathematical ideas in the first framing. In the second framing, he provided two additional mathematical ideas and elaborated on each of them.

In P77's first explanation, he used probability to explain the way the virus spreads, and a tree diagram is added as an image to exemplify the way it spreads. In the second framing, P77 used the idea of acceleration to emphasize that the number of infected people grows rapidly and used an image of a function without a limit, which might imply that he meant a monotonically increasing function, similar to an exponential function. However, P77 also referred to an extremum point of the function and a shift in the function's direction. Here, it seems that he tried to model a situation wherein the number of infected people could not increase indefinitely because the population is limited. P77's idea of having an extremum point (maximum) followed by a decreased rate of change is similar to the first derivative function of the logistic curve (see Fig. 2). This idea might have led him to suggest the quadratic function as an image. It could be that he was picturing in his mind a function that has one maximum extremum, which may have led him to this kind of generalization.

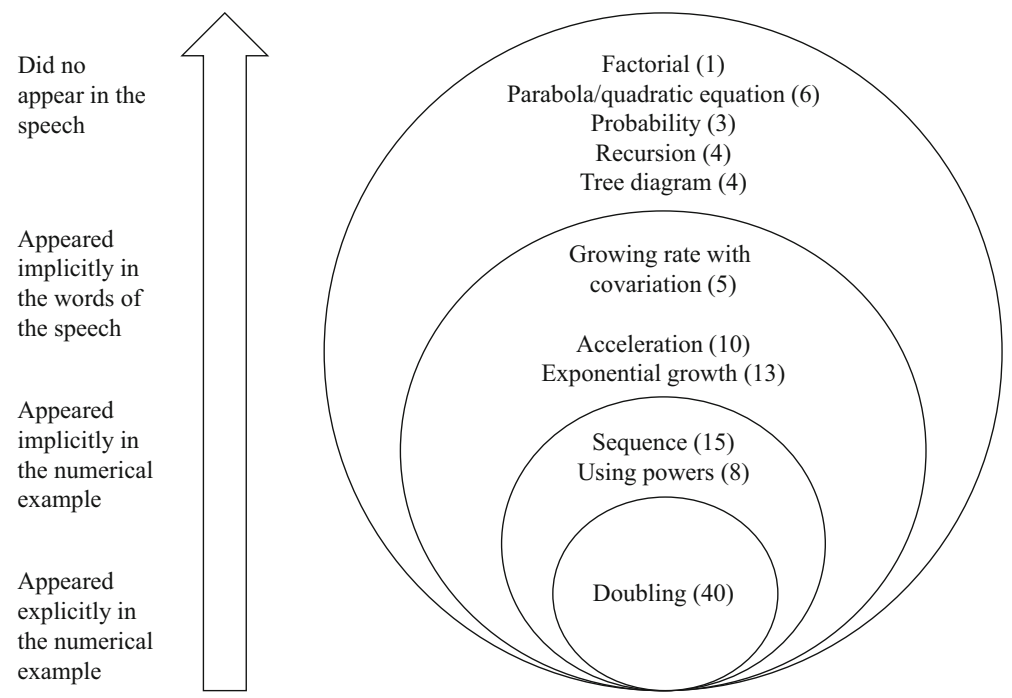

Fig. 8 Organization of the 11 mathematical ideas that appears in the second framing according to the extent of their presence in the PM's speech. The number of appearances is in brackets $(N=109)$ 
The variation of mathematical ideas in the second framing could result from the framing itself, i.e., the request to explain the PM's speech. Figure 8 presents the 11 mathematical ideas according to the extent of their presence in the PM' speech, those explicitly mentioned in the numerical example, those implicitly suggested by the numerical example, those implicitly suggested by the wording of his speech, and those that did not appear in the speech at all. The number of appearances of each mathematical idea used in the graduates' explanations appears in brackets $(N=109)$ and refers to the second framing.

The idea of doubling did not emerge in the first framing (see Table 5), only in the second framing, implying a possible effect of the PM's numerical example. The words "... one person infects two people. Each one infects two more. Four infect eight... and so forth..." probably led to the idea of doubling. Therefore, this idea was prominent in participants' responses.

The ideas of using powers and sequence were not mentioned explicitly in the speech but could be inferred from the numerical example used. Using powers emerged only in the second framing, whereas sequence appeared somewhat in the first framing and to a larger extent in the second framing.

The ideas of exponential growth, growing rate with covariation, and acceleration were not mentioned explicitly in the speech but could be inferred from the wording used. The idea of exponential growth could be inferred from the words "geometric series." Acceleration might be inferred from, "First it spreads slowly and then at tremendous speed...." Furthermore, the PM referred to time implicitly in two quantities that change simultaneously-time and the number infected people - which could explain the participants' reference to a growing rate with covariation.

The other five ideas were not implied by the words the PM used in his speech and appeared in small numbers. Their emergence could result from the participants' associated connection, as articulated by P49, "It also reminds me a bit of the factorial in mathematics." Or by P22, "I don't remember how it is connected or what exactly the recursion method is; simply, this is what came to my mind." Furthermore, the ideas of the tree diagram and recursion appear in the literature dealing with exponents as representations of an exponential function (Confrey \& Smith, 1995), which could be further evidence of the associated connections participants drew. These ideas appear in the last circle in Fig. 8.

We see that, in general, there is some connection between the extent of explicitness of the mathematical ideas within the PM's speech and their appearance within the participants' explanations in the second framing. Still, some mathematical ideas could not be accounted for through the explicitness of the idea in the speech. Consequently, we explored the participants' explanations in each of the two questionnaire sections in relation to their school backgrounds, in particular, the level at which they studied high school mathematics and the subjects they majored in.

\subsection{Relations of the participants' explanations and their school backgrounds}

The results of the exploration of participants' explanations given in each of the two framings (the questionnaire's two sections) in relation to their school backgrounds are presented in Fig. 9. The figure is divided into the two framings. The first framing, on the left-hand side, includes the theme of the explanation (i.e., based on biology, social sciences, mathematics, or no explanation) and the majors and the participants' corresponding mathematics level. For clarification, we will note again that a participant could have one or two majors so summing up the number of majors in the first framing exceeds the number of participants. On the right- 


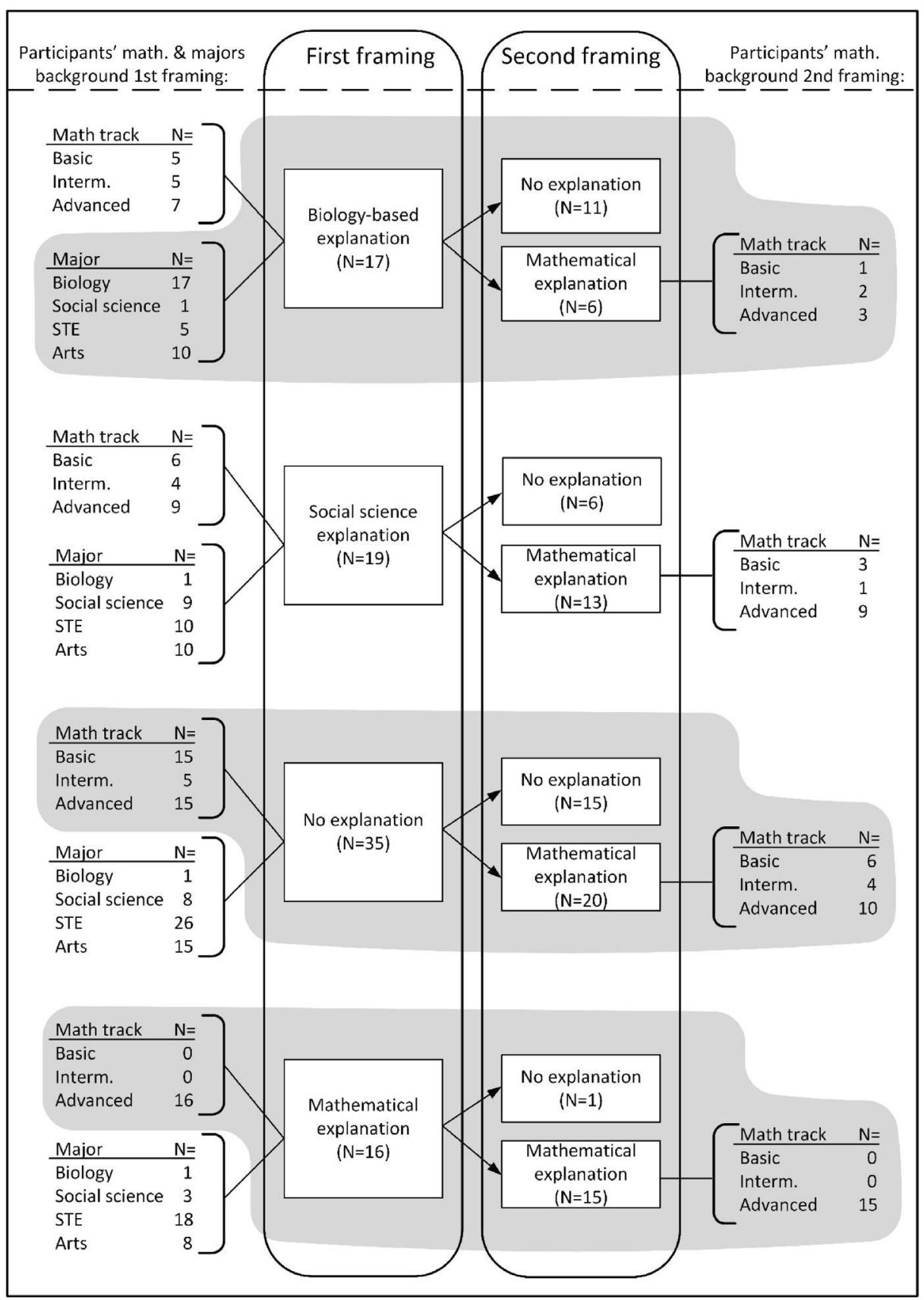

Fig. 9 Types of explanation in each framing in relation to the participants' school background

hand side, the second framing is noted by a mathematical explanation and no explanation. For each explanation in the second framing, the participants' level of mathematics is noted in the same way. 
As shown in Fig. 9, in the first framing, where no specific request for mathematical explanations exists, 19 participants' responses (out of 87) were based on social science issues. For example, P11 wrote: "In civics class, we learned about globalization, which describes the world becoming a 'global village' in various aspects of life. It helps me understand why the virus that spread in China spread into all to the countries of the world in less than a month." Seventeen participants used biology-based explanations, as illustrated in P2's explanation (Table 3). Sixteen used mathematical explanations, as illustrated in P77's explanation (Table 6). Thirty-five participants gave no explanation in the first framing. As shown in Fig. 7, in the second framing, in which participants were requested to explain the PM's speech, which described the spread of the coronavirus as a geometric series, 73 participants addressed mathematical explanations, while 14 included no other type of explanation.

Figure 9 further presents relations between the shifts in explanations between framings for each participant and his/her school background, i.e., their mathematics track level and major fields of study. The figure shows several interesting patterns. First, all 16 participants who used mathematical explanations in the first framing had studied mathematics in the advanced track. Fifteen of them added even more mathematical ideas in the second framing.

Second, all 17 participants who used biology-based explanations in the first framing had majored in high school biology, with the majority of them (11 participants) also studying in the advanced or intermediate track in mathematics. Six of the 17 switched to a mathematical explanation in the second framing. Similarly, the 19 participants who used social sciencebased explanations in the first framing had all majored in social sciences or arts and humanities in high school. The majority (13 participants) offered a mathematical explanation in the second framing.

Third, 15 out of the 26 participants who had studied basic-level mathematics did not offer any explanation at all in the first framing. The majority of them ( 9 of the 15) did not offer any explanation in the second framing either. Fifteen (out of 47) participants who studied highlevel mathematics offered no explanation in the first framing. However, ten of the 15 offered a mathematical explanation in the second framing.

These patterns suggest possible relations between the types of explanations elicited in the first section of the questionnaire and the participants' school background in terms of both their majors and the level of mathematics they studied. This is further discussed in the following section.

\section{Discussion and conclusion}

In this study, analysis of participants' mathematical explanations for the spread of the coronavirus indicated a collection of mathematical ideas. Here, we will discuss possible sources for the appearance of the ideas and suggest directions for further investigation.

The ideas of doubling, using powers, sequence, exponential growth, and growing rate with covariation are integral to the context of the spread of the virus. Doubling, using powers, and sequence were mentioned explicitly or implicitly in the numerical example used in the PM's speech. Exponential growth, which is a part of the Israeli curriculum in all levels of mathematics, is implied from the PM's use of geometric series. Growing rate with covariation serves as key concept in learning exponential growth in a realistic setting (Ellis et al., 2016). Additionally, the idea of tree diagram and recursion, which were not mentioned in the PM's speech, are mentioned in the literature among exponential growth representations (Confrey \& 
Smith, 1995). This research was conducted under the assumption that the spread of COVID-19 can be explained using exponential growth, which is deeply embedded within the Israeli curriculum. Therefore, we did not expect the results to include the ideas of probability, parabola, quadratic function, acceleration, and factorial. We found it interesting that participants used these ideas to explain the spread of COVID-19. Probability can seem as an additional explanation for the spread of the virus, from a different mathematical perspective. Parabola and quadratic function can emerge as participants try to model the spread of the virus and while picturing in their mind a graph that increases and then decreases - parabola might be the concept that comes to their mind. Acceleration might be referred to here not in the sense of the change in velocity but more in the form of everyday language, which is even apparent in the PM's speech. An interesting follow-up might be to ascertain, using an in-depth, interviewbased methodology, the ways in which the mathematical ideas that emerged in this study serve the participants as they explain the spread of the virus. The participants could explain the way in which these ideas emerge and explain their perspectives.

In addition to the participants' mathematical explanations for the spread of COVID-19, this study also presents linkage between the mathematics-based, biology-based, and social sciencebased explanations and the participants' chosen majors in high school. We suggest that the construct of intuitive knowledge offered by Kapon (2016) that "accounts for how things are" (p. 169) while relying on the experience with the world, education, language, interaction, and framing can explain the diverse explanations, mathematical and nonmathematical alike. This suggestion is supported firstly by the connection drawn between the participants' explanations and the framing of the PM's speech. As mentioned in the findings section, the idea of doubling was introduced only after participants were requested to explain the speech (Table 5) and secondly, the participants' multiple phrases that suggested intuitive notions, such as "I believe that this function..." (P77) or "it also reminds me a bit of the factorial" (P49). Thirdly, it is supported by findings that indicate that participants' mathematical explanations can be linked to the level of high school mathematics they had studied. In the first framing, in which participants were asked to explain the spread of the coronavirus in terms of their high school studies, with no restriction to mathematics, all those participants who used mathematical explanations had studied mathematics in the advanced track. The participants who did not offer mathematical explanations, even in the second framing, were those who had studied basic-level mathematics. Furthermore, biology majors offered biology-based explanations, whereas social sciences or arts and humanities majors offered social science-based explanations.

In this study, we chose to use Kapon's (2016) framework. However, we did not differentiate between intuitive knowledge and mechanism. Our data collection method, the online questionnaire, did not invite respondents to answer at length, and the researchers were not present to prompt and ask for additional explanations. After receiving the data, there were almost no opportunities for the researchers to elicit further details - a fact that might have caused participants to elaborate on the mechanism in which COVID-19 spread. Nevertheless, this study augments the few existing studies in mathematics education that did use similar frameworks based on that of diSessa (e.g., 1996) and were guided by the framework's ability "to highlight diverse, fine-grained knowledge resources that a person could draw upon" (e.g., Izsák \& Jacobson, 2017; p.301). These studies were conducted in several mathematical fields, including multiplication and probability (e.g., Izsák, 2005; Izsák \& Jacobson, 2017; Moschkovich, 1998; Wagner, 2006), but to the best of our knowledge, not in making sense 
of realistic phenomena such of the spread of the coronavirus. Beyond strengthening the potential of implementing such frameworks in mathematics education (e.g., diSessa, 1996), the present study exposes the intuitive knowledge that Israeli high school graduates drew on when trying to make sense of a realistic phenomenon, which they observe and experience daily, through the mathematics that they learned in school. Thus, this study lays the groundwork for the "range of knowledge resources" (Izsák \& Jacobson, 2017; p. 335) on sensemaking of realistic phenomena through mathematics and calls for further research, in different contexts and with different populations, to extend the knowledge in this field.

From a practical perspective, we suggest that when teaching exponential growth, teachers could consider the set of intuitive ideas that emerged in this study, both the nonmathematical explanations and the intuitive mathematical explanations which include parabola, quadratic function, and acceleration. Furthermore, we see this exploration of explaining a phenomenon through mathematics as an exciting direction for future implementation. We embrace Li and Schoenfeld's (2019) suggestion to adopt the practice of sense-making, explaining the world's phenomena, as a possible shift for mathematics education: "Conceiving mathematics as making sense should help promote conceptual changes in mathematical practice to value idea generation and design activity. Connections generated from such a shift will support teaching and learning not only in individual STEM disciplines but also in integrated STEM education.” (p. 7).

Acknowledgements The first author, Sigal-Hava Rotem, is grateful to the Azrieli Foundation for the award of an Azrieli Fellowship.

\section{References}

Ayalon, M., \& Hershkowitz, R. (2018). Mathematics teachers' attention to potential classroom situations of argumentation. The Journal of Mathematical Behavior, 49, 163-173.

Baltar, F., \& Brunet, I. (2012). Social research 2.0: Virtual snowball sampling method using Facebook. Internet Research, 22(1), 57-74.

Batista, M. (2020). Estimation of the final size of the COVID-19 epidemic. MedRxiv, 10, 16-20023606.

Carraher, T. N., Carraher, D. W., \& Schliemann, A. D. (1985). Mathematics in the streets and in schools. British Journal of Developmental Psychology, 3(1), 21-29.

Castillo-Garsow, C. (2012). Continuous quantitative reasoning. In R. Mayes \& L. Hatfield (Eds.), Quantitative reasoning and mathematical modeling: A driver for STEM integrated education and teaching in context, WISDOMe monographs (vol. 2, pp. 55-73). University of Wyoming Press.

Castillo-Garsow, C., Johnson, H. L., \& Moore, K. C. (2013). Chunky and smooth images of change. For the Learning of Mathematics, 33(3), 31-37.

Confrey, J., \& Smith, E. (1995). Splitting, covariation, and their role in the development of exponential functions. Journal for Research in Mathematics Education, 26(1), 66-86.

Cramer, J. S. (2002). The origins of logistic regression. Tinbergen Institute Discussion Paper, No. 02-119/4, Tinbergen Institute, Amsterdam and Rotterdam

Davis, J. D. (2009). Understanding the influence of two mathematics textbooks on prospective secondary teachers' knowledge. Journal of Mathematics Teacher Education, 12(5), 365-389.

diSessa, A. A. (1996). What do "just plain folk" know about physics. In D. Olson \& N. Torrance (Eds.), The handbook of education and human development (pp. 709-730). Blackwell publishers.

Doerr, H. M. (2006). Examining the tasks of teaching when using students' mathematical thinking. Educational Studies in Mathematics, 62(1), 3-24.

Ellis, A. B., Ozgur, Z., Kulow, T., Dogan, M. F., \& Amidon, J. (2016). An exponential growth learning trajectory: Students' emerging understanding of exponential growth through covariation. Mathematical Thinking and Learning, 18(3), 151-181.

Fischbein, E. (1987). Intuition in science and mathematics: An educational approach. Reidel.

Geva, Y., \& Tal, R. (2014). Mathematics questionnaire no. 802. Three-point track. Yoel Geva. 
Hoyles, C., Noss, R., \& Pozzi, S. (2001). Proportional reasoning in nursing practice. Journal for Research in Mathematics Education, 31(1), 4-27.

Izsák, A. (2005). "You Have to Count the Squares": Applying knowledge in pieces to learning rectangular area. The Journal of the Learning Sciences, 14(3), 361-403.

Izsák, A., \& Jacobson, E. (2017). Preservice teachers' reasoning about relationships that are and are not proportional: A knowledge-in-pieces account. Journal for Research in Mathematics Education, 48(3), 300-339.

Kapon, S. (2016). Unpacking sensemaking. Science Education, 101(1), 165-198.

Kapon, S., \& diSessa, A. A. (2012). Reasoning through instructional analogies. Cognition and Instruction, 30(3), 261-310.

Li, Y., \& Schoenfeld, A. H. (2019). Problematizing teaching and learning mathematics as 'given' in STEM education. International Journal of STEM Education, 6, 44. https://doi.org/10.1186/s40594-019-0197-9

Lipkin, L., \& Smith, D. (2001). Logistic growth model. JOMA, 1(3) https://www.maa.org/press/periodicals/loci/ joma/logistic-growth-model-introduction

Mayer, R. E. (1982). Memory for algebra story problems. Journal of Educational Psychology, 74(2), $199-216$.

Morais, A. F. (2020). Logistic approximations used to describe new outbreaks in the 2020 COVID-19 pandemic. arXiv preprint arXiv:2003.11149.

Moschkovich, J. N. (1998). Resources for refining mathematical conceptions: Case studies in learning about linear functions. The Journal of the Learning Sciences, 7(2), 209-237.

Reed, L. J., \& Pearl, R. (1927). On the summation of logistic curves. Journal of the Royal Statistical Society, 90(4), 729-746.

Tang, Y., \& Hew, K. F. (2017). Is mobile instant messaging (MIM) useful in education? Examining its technological, pedagogical, and social affordances. Educational Research Review, 21, 85-104.

Thompson, P. W. (2011). Quantitative reasoning and mathematical modeling. In L. L. Hatfield, S. Chamberlain, \& S. Belbase (Eds.), New perspectives and directions for collaborative research in mathematics education. University of Wyoming Press.

Thompson, P. W., \& Carlson, M. P. (2017). Variation, covariation, and functions: Foundational ways of thinking mathematically. In J. Cai (Ed.), Compendium for research in mathematics education (pp. 421-456). National Council of Teachers of Mathematics.

Van den Heuvel-Panhuizen, M., \& Drijvers, P. (2014). Realistic mathematics education. In S. Lerman (Ed.), Encyclopedia of mathematics education (pp. 713-717). Springer Nature.

Wagner, J. F. (2006). Transfer in pieces. Cognition and Instruction, 24(1), 1-71. https://doi.org/10.1207/ s1532690xci2401 1

Webb, D. C., Van der Kooij, H., \& Geist, M. R. (2011). Design research in the Netherlands: Introducing logarithms using realistic mathematics education. Journal of Mathematics Education at Teachers College, 2(1), 47-52.

Wu, K., Darcet, D., Wang, Q., \& Sornette, D. (2020). Generalized logistic growth modeling of the COVID-19 outbreak in 29 provinces in China and in the rest of the world. arXiv preprint arXiv:2003.05681.

Publisher's note Springer Nature remains neutral with regard to jurisdictional claims in published maps and institutional affiliations. 